\title{
Efficacy and safety of left atrial appendage occlusion in atrial fibrillation patients with chronic kidney disease: a systematic review and meta-analysis
}

\author{
Hai-Fu Zhang ${ }^{1,2, \dagger}$, Qin-Xia Zhang ${ }^{2, \dagger}$, Yuan-Yuan Zhang ${ }^{1}$, Dong Yang ${ }^{1}$, Zhao Xu ${ }^{1}$, Qi-Bin Jiao ${ }^{1,2}$ and Xing-Wei Zhang ${ }^{1,2, *}$ \\ ${ }^{1}$ Division of Cardiology, The Affiliated Hospital of Hangzhou Normal University, Hangzhou 310015, P. R. China \\ ${ }^{2}$ School of Medicine, Hangzhou Normal University, Hangzhou 311121 , P. R. China \\ ${ }^{*}$ Correspondence: xwzhang@hznu.edu.cn (Xingwei Zhang) \\ †These authors contributed equally.
}

DOI: $10.31083 /$ i.rcm.2020.03.62

This is an open access article under the CC BY 4.0 license (https://creativecommons.org/licenses/by/4.0/).

\begin{abstract}
Atrial fibrillation (AF) is the most common arrhythmia among the elderly, and more frequently occur in those with chronic kidney disease (CKD). Left atrial appendage occlusion (LAAO) is used as a mechanical alternative approach for prevention of AF-related thromboembolisms. This meta-analysis was conducted to provide suggestions for the clinical application of LAAO in AF patients with CKD. The incidence of perioperative adverse events and other clinical effects after operation was by a single rate meta-analysis. Results showed that incidence of adverse events in the perioperative period after LAAO was generally low, with only pericardial effusion / tamponade $(1.90 \%)$ and mortality rate (1.10\%). During the follow-up period, the incidence of stroke/transient ischemic attack (TIA) and bleeding were $2.17 \%$ and $4.53 \%$, respectively. A low incidence rate of adverse events was found in the perioperative period following LAAO. These results indicate that LAAO more effectively prevents the occurrence of stroke/TIA and minimizes bleeding events than oral anticoagulants.
\end{abstract}

\section{Keywords}

Atrial fibrillation; chronic kidney disease; left atrial appendage occlusion

\section{Introduction}

Atrial fibrillation (AF) is the most common cardiac rhythm disorder, with a prevalence of $1-2 \%$ in the general population (Dalia et al., 2020). AF patients have a 3-5 times higher risk of ischemic stroke relative to healthy individuals (Malyszko et al., 2018). Moreover, AF associated cardiogenic cerebral infarction has high recurrence rate and poor prognosis (Akagi et al., 2019).

The global incidence rate of adult chronic kidney disease (CKD) is about $13.4 \%$ (Noble and Taal, 2019). Relative to the general population, arrhythmias are considerably higher in CKD patients with a prevalence rate in the range of 19-24\% (Malyszko et al., 2018). AF and CKD have common risk factors, and both are associated with increased risk of thromboembolism, stroke, car- diovascular morbidity and mortality (Coleman et al., 2019; Potpara et al., 2018).

Oral anticoagulants reduce the risk of stroke by two-thirds in AF patients (Mtwesi and Amit, 2019). However, oral anticoagulants undergo varying degrees of kidney clearance, and are associated with a higher risk of bleeding in CKD patients (Shin et al., 2018). In AF patients, most of the thrombus forms in the left atrial appendage. Thus, left atrial appendage occlusion (LAAO) has emerged as a mechanical alternative intervention to prevent AF-related thromboembolisms (Kreidieh et al., 2015). Patients fitted with LAAO devices do not require prolonged oral anticoagulants, but may only need single antiplatelet therapy or no therapy (Boersma et al., 2019). Such patients consistently exhibit low rates of stroke and major bleeding events. Therefore, LAAO is suitable for patients contraindicated for oral anticoagulants, end-stage renal disease, recurrent gastrointestinal hemorrhage, and prior intracranial hemorrhage (Black-Maier et al., 2019). Here, we performed a meta-analysis to determine the efficacy and safety of LAAO in CKD patients with AF.

\section{Methods}

\subsection{Search strategy and eligibility criteria}

This systematic review and meta-analysis adhered to preferred reporting items for systematic reviews and meta-analyses guidelines (PRISMA) (Shamseer et al., 2015), and was performed in line with a prespecified protocol. Two investigators (Haifu Zhang and Qinxia Zhang) searched the PubMed, Google scholar, Web of Science, Cochrane Central Register of Controlled Trials (CENTRAL), and OVID to identify relevant studies. The following key medical subject headings (MeSH) terms and Emtree terms were used: atrial fibrillation, atrial appendage, kidney OR renal, and oral anticoagulants. The search was restricted to randomized controlled trials (RCTs) and clinical research published from inception up to April 1, 2020. Reference lists of selected studies and previous reviews were manually screened to identify potential eligible trials. Case reports, editorials, expert opinions, review articles, editorials, guidelines, and non-English studies were excluded.

$\mathrm{CKD}$ is defined as an estimated glomerular filtration rate 
Table 1. Characteristics of 7 studies included in the review

\begin{tabular}{|c|c|c|c|c|c|c|c|c|c|}
\hline Study & Study design & Age & Male (\%) & $\begin{array}{c}\text { CHADS-VASc/ } \\
\text { CHADS }\end{array}$ & HAS-BLED & $\begin{array}{l}\text { Treatment group } \\
\text { (n) }\end{array}$ & Renal function & $\begin{array}{c}\text { Previous } \\
\text { Stroke/ } \\
\text { TIA (\%) }\end{array}$ & $\begin{array}{c}\text { Previous } \\
\text { Major } \\
\text { Bleeding }(\%)\end{array}$ \\
\hline Luani et al. (2019) & cohort study & $75.9 \pm 6.7$ & 57.5 & $4.5 \pm 1.4$ & $3.6 \pm 1.0$ & Watchman: 73 & $\begin{array}{l}\mathrm{eGFR}<60 \\
\mathrm{~mL} / \mathrm{min}\end{array}$ & 16.4 & I \\
\hline Kefer et al. (2016) & RCT & $77.9 \pm 7.3$ & 54.7 & $4.9 \pm 1.5$ & $3.4 \pm 1.3$ & ACP: 375 & $\begin{array}{l}\mathrm{eGFR}<60 \\
\mathrm{~mL} / \mathrm{min}\end{array}$ & 32.3 & 49.1 \\
\hline Brockmeyer et al. (2019) & cohort study & $78.2 \pm 7.3$ & 49.4 & $4.7 \pm 1.3$ & $3.9 \pm 0.9$ & $\begin{array}{l}\text { ACP: } 61 \text { Watch- } \\
\text { man: } 5 \text { Am- } \\
\text { platzer } \\
14\end{array}$ & $\begin{array}{l}\mathrm{eGFR}<60 \\
\mathrm{~mL} / \mathrm{min}\end{array}$ & 17.3 & 39.6 \\
\hline Genovesi et al. (2018) & $\begin{array}{l}\text { prospective } \\
\text { cohort study }\end{array}$ & $71.8 \pm 9.6$ & 76 & $4.0 \pm 1.5$ & $4.4 \pm 0.9$ & $\begin{array}{l}\text { Watchman: } \\
24 \quad \text { Amplatzer } \\
\text { Amulet: } 26\end{array}$ & $\begin{array}{l}\text { eGFR }<15 \\
\mathrm{~mL} / \mathrm{min}, \\
\text { dialysis }\end{array}$ & on & 66 \\
\hline Xue et al. (2018) & $\mathrm{RCT}$ & $77.0 \pm 7.2$ & 60.9 & $4.3 \pm 1.5$ & $4.0 \pm 1.0$ & Watchman: 151 & $\begin{array}{l}\mathrm{eGFR}<60 \\
\mathrm{~mL} / \mathrm{min}\end{array}$ & 11.3 & 25.8 \\
\hline So et al. (2018) & $\begin{array}{l}\text { retrospective } \\
\text { study }\end{array}$ & $72 \pm 8$ & 65.3 & $5.1 \pm 1.5$ & $3.4 \pm 0.1$ & ACP/Amulet: 71 & $\begin{array}{l}\mathrm{eGFR}<60 \\
\mathrm{~mL} / \mathrm{min}\end{array}$ & I & I \\
\hline Della Rocca et al. (2018) & cohort study & $77 \pm 7$ & 46.1 & $4.9 \pm 1.8$ & $3.7 \pm 0.9$ & 104 & $\begin{array}{l}\mathrm{eGFR}<60 \\
\mathrm{~mL} / \mathrm{min}\end{array}$ & I & I \\
\hline
\end{tabular}

NOTE: RCT: randomized controlled trial; eGFR: estimated glomerular filtration rate; TIA: transient ischemic attack.

(eGFR) of $<60 \mathrm{~mL} / \mathrm{min} / 1.73$ for 3 months or more, irrespective of the cause (Tomson and Duffy, 2019). Based on eGFR (Levey et al., 2011), CKD is staged as follows: stage 3a: $45-59 \mathrm{~mL} / \mathrm{min} / 1.73$ $\mathrm{m}^{2}$, stage $3 \mathrm{~b}: 30-44 \mathrm{~mL} / \mathrm{min} / 1.73 \mathrm{~m}^{2}$, stage $4: 15-29 \mathrm{~mL} / \mathrm{min} / 1.73$ $\mathrm{m}^{2}$, stage $5:<15 \mathrm{~mL} / \mathrm{min} / 1.73 \mathrm{~m}^{2}$ or on dialysis. In LAAO trials for $\mathrm{AF}$, only those that included adult patients ( $>18$ years old), used LAAO for AF and subjects with CKD at eGFR $<60 \mathrm{~mL} / \mathrm{min}$ or undergoing dialysis were included.

\subsection{Data abstraction and end points}

Two independent investigators (Haifu Zhang and Qinxia Zhang) extracted the following data from the included studies: authors, year of publication, and baseline features, including renal function, previous stroke/transient ischemic attack (TIA), previous major bleeding, congestive heart failure and hypertension. Incidence of adverse events during the perioperative and follow-up periods were analyzed.

\subsection{Quality assessment}

Quality of included studies was evaluated using the Cochrane Collaboration's randomized trial bias risk assessment tool. Trials were categorized as having low, unclear, or high bias risks in the following domains: random sequence generation allocation, allocation concealment, blinding of participants and personnel, blinding of outcome assessment, incomplete outcome data, selective reporting and other bias. Quality was independently assessed by 2 investigators (Haifu Zhang and Qinxia Zhang) and any discrepancy was resolved through consensus. All eligible trials were included in the meta-analysis regardless of quality level.

\subsection{Statistical analysis}

The extracted data were analyzed using Microsoft Excel 2016 and Revman 5.3. The primary outcomes were the incidence of adverse events during the perioperative and follow-up periods. A meta-analysis of single-group rates was conducted to analyze the extracted data. When an event was 0 , it was corrected to 0.5 to avoid miscalculation. The inverse-variance method and randomeffect approach were employed to analyze dichotomous data. $P$ $\leq 0.05$ indicated research heterogeneity. $\mathrm{I}^{2}$ values of 25,50 and $75 \%$ indicated low, medium and high degree of heterogeneity, respectively.

\section{Results \\ 3.1 Search results}

Our literature search retrieved 1829 relevant reports, of which 7 involved the use of LAAO in AF patients with CKD (Fig. 1). After applying the inclusion and inclusion criteria, 905 patients treated with LAAO, of which 67 were under hemodialysis were included in the final analysis. In all patient, mean score of CHADSVASc/CHADS exceeded 4, and mean score of HAS-BLED exceeded 3.5, with high risks of thromboembolic and bleeding. The follow-up period was 1-2 years (Table 1). The quality of included trails is shown in Fig. 2. CKD patients fitted with LAAO devices exhibited improved clinical features during follow-up with low incidence of cardiogenic mortality, bleeding and stroke.

\subsection{Periprocedural complications}

To determine the incidence of various adverse events in the perioperative period of LAAO, the incidence of perioperative death, stroke/TIA, bleeding and pericardial effusion/tamponade were calculated. In general, we found a low rate of adverse events in the perioperative period, and renal function was not affected. After LAAO, the main adverse event was pericardial effusion/tamponade, occurring in 13 of the 670 patients, representing an incidence rate of $1.90 \%$ (OR:0.02, 95\% CI: 0.00-0.10). The incidence of bleeding and stroke/TIA in the perioperative period was 1.60\% (OR:0.02, 95\% CI: 0.01-0.13) and 1.40\% (OR:0.02, 95\%CI: 


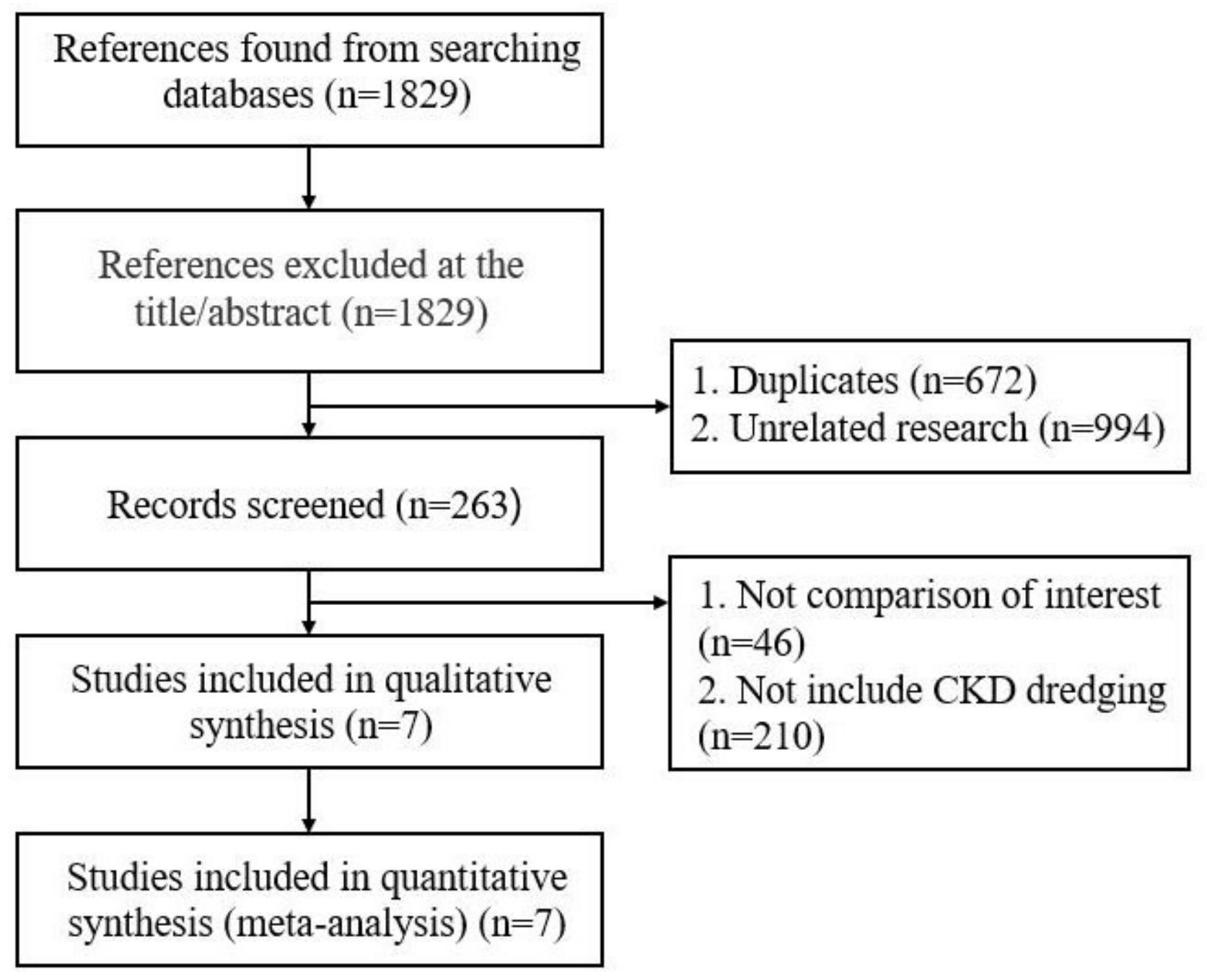

Fig. 1. Preferred reporting items for systematic reviews and meta-analyses flow diagram. Using different keywords, a total of 1829 articles were retrieved. Through reading title, abstract and full-text, there were 7 relevant articles finally included in our meta-analysis. CKD: chronic kidney disease.

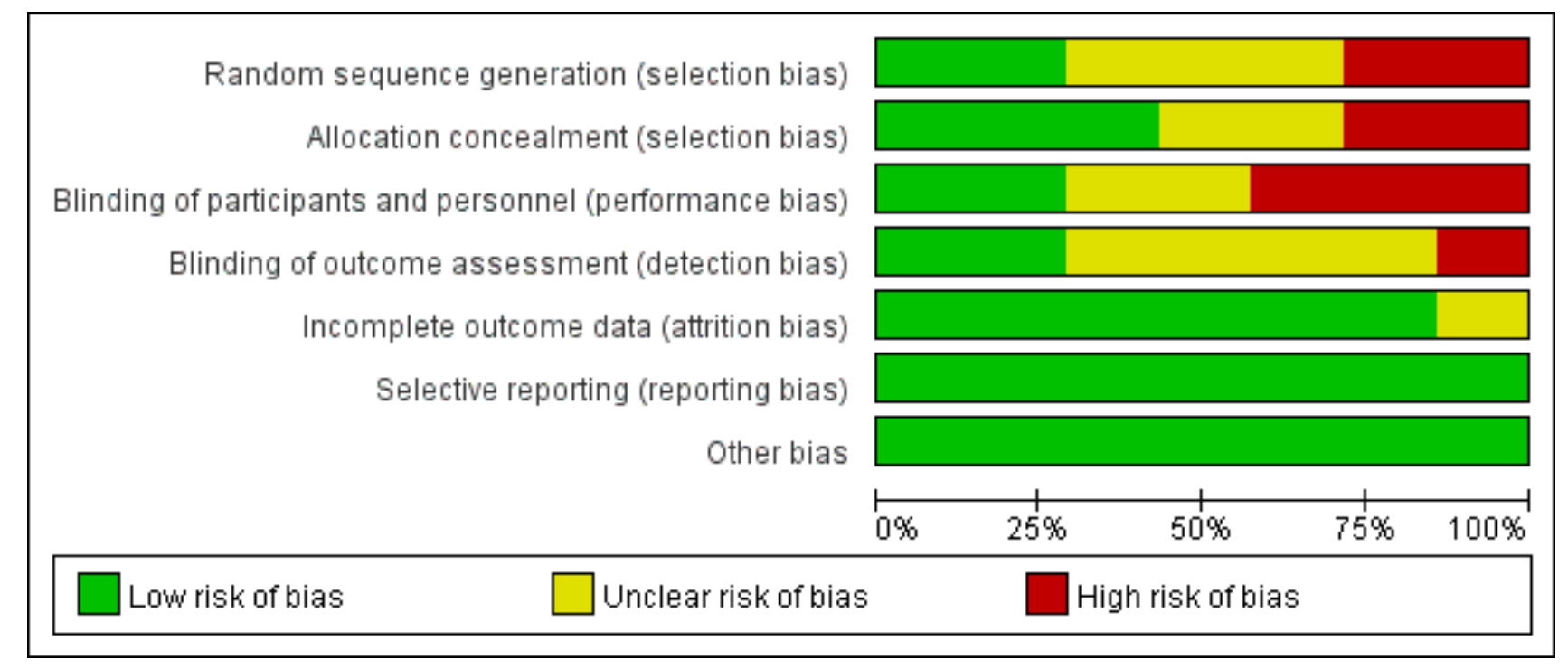

Fig. 2. Quality evaluation char. Select bias and performance bias were higher because of the inclusion of non-randomized controlled trial. 


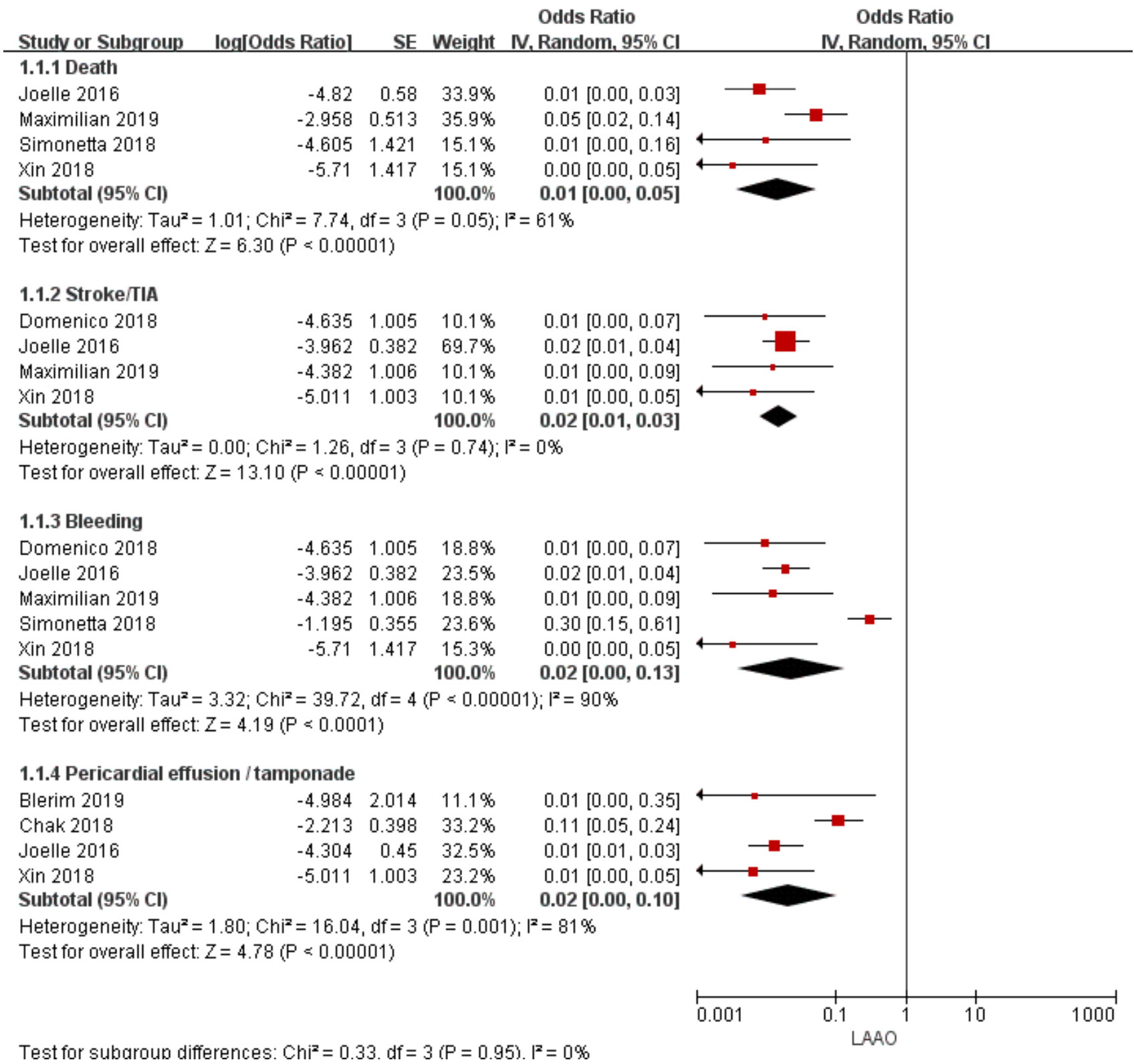

Fig. 3. Forest plots showing the effect of clinical outcomes on the overall risk of perioperative death, stroke/ transient ischemic attack, bleeding, pericardial effusion/tamponade. A total of 7 studies were included. During the perioperative period, mortality rate was $1.10 \%$ (OR:0.01, 95\%CI: 0.00-0.05). The incidence of stroke/TIA and bleeding in the perioperative period was $1.40 \%$ (OR:0.02, $95 \% \mathrm{CI}: 0.01-0.03)$ and $1.60 \%$ (OR:0.02,95\%CI: $0.00-0.13$ ). The pericardial effusion/tamponade rate was $1.90 \%$ (OR:0.02, $95 \%$ CI: $0.00-0.10$ ).

0.01-0.03), respectively. Seven patients died during the perioperative period, representing a mortality rate of $1.10 \%$ (OR:0.01, 95\% CI: 0.00-0.05) (Fig. 3).

\subsection{Follow-up}

The occurrence of adverse events during postoperative and follow-up periods was reported in most clinical trials. Notably, 55 among 448 patients died after LAAO treatment. Moreover, allcause mortality of AF combined with CKD was $12.28 \%$ (OR:0.12, 95\% CI: 0.09-0.17), while the cardiogenic mortality rate was $3.98 \%$ (OR:0.04, 95\%CI: 0.03-0.07). Oral anticoagulants are not needed after LAAO,hence the incidence of bleeding was $4.53 \%$ (OR:0.04, 95\%CI: 0.02-0.07). LAAO exhibited high safety, with a low risk of stroke/TIA during follow-up which was $2.17 \%$ (OR:0.03, 95\%CI: 0.02-0.05) (Fig. 4).

\subsection{CKD vs. Non-CKD patients}

The results of follow-up after LAAO in CKD versus non-CKD patients were only reported in 2 trials. Patients with CKD exhibited higher all-cause mortality rate after undergoing LAAO relative to those without CKD (12.93\% vs. $6.54 \%$; RR:2.00; 95\%CI: 1.09-3.68, $P<0.05)$. There were no significant differences in stroke/TIA (RR: 0.52, $P=0.24$ ), bleeding (RR: $1.02, P=0.96$ ) and cardiac death (RR: $1.34, P=0.65)$ between $\mathrm{CKD}$ versus nonCKD patients (Fig. 5). 


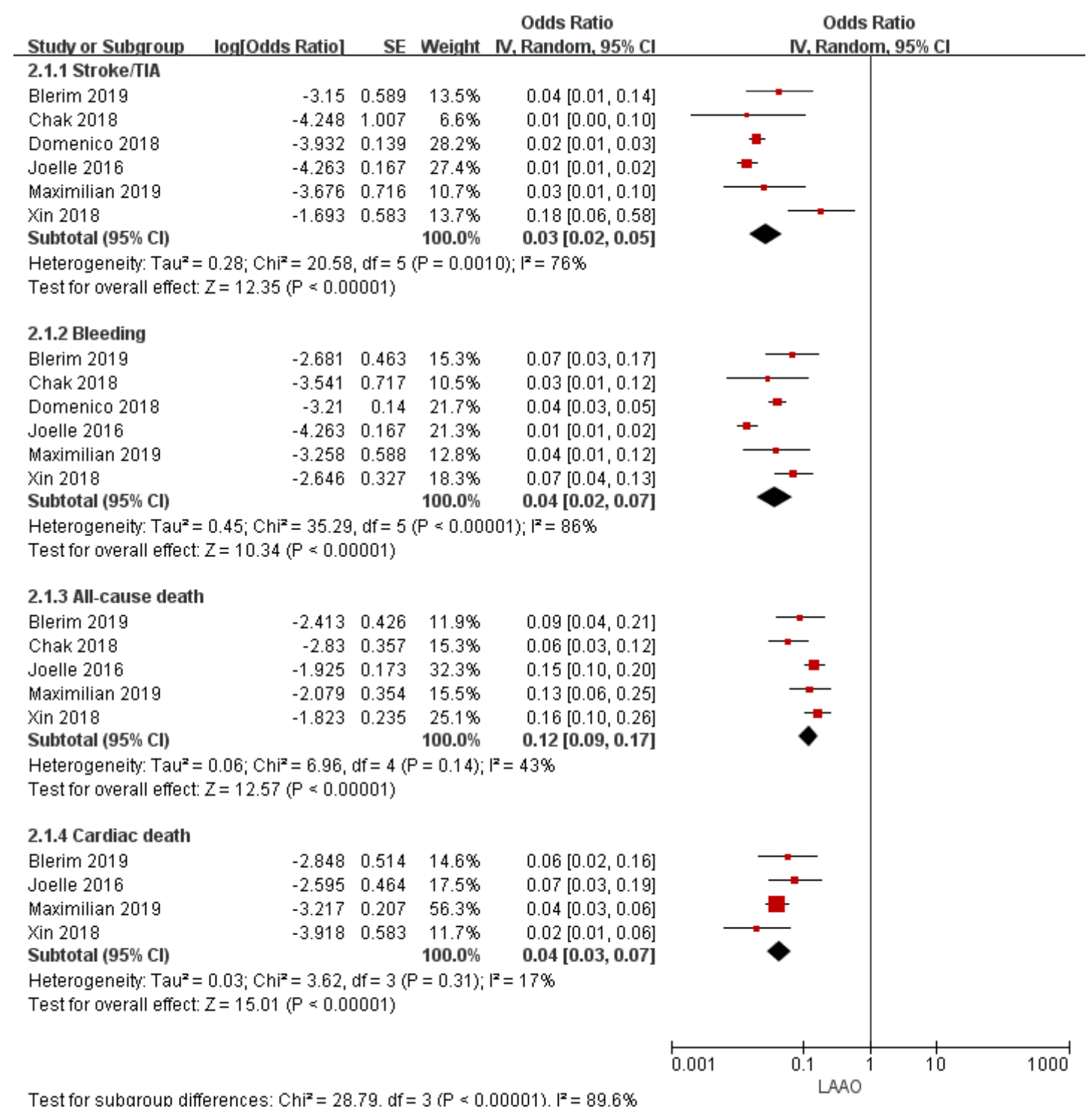

Fig. 4. A forest plot showing the effect of left atrial appendage occlusion on the overall risk of stroke/ transient ischemic attack, bleeding, all-cause death and cardiogenic death in the follow up time. A total of 6 studies were included. The incidence of stroke/TIA and bleeding in the follow up time was 2.17\% (OR:0.03, 95\%CI: 0.02-0.05) and 4.53\% (OR:0.04, 95\%CI: 0.02-0.07). All-cause mortality was 12.28\% (OR:0.12, 95\%CI: 0.09-0.17), and the cardiogenic mortality rate was $3.98 \%$ (OR:0.04, 95\% CI: $0.03-0.07$ ) during follow-up.

\section{Discussion}

This meta-analysis was conducted on 7 studies reporting on the use of LAAO in AF patients with CKD. The main devices used were Watchman, ACP, and Amplatzer-Amulet. Single-rate metaanalysis revealed that incidence of perioperative adverse events was $1-2 \%$, indicating good perioperative safety of LAAO. A low incidence of adverse events was also reported during follow-up. No significant differences in stroke/TIA, bleeding and cardiac death incidence were observed between patients with CKD and non-CKD patients.

\subsection{Perioperative safety}

Early use of LAAO was associated with low surgical success rate (about $90 \%$ ) and high perioperative complication rates (about $8.4 \%$ ) (Holmes et al., 2009). However, advances in surgical techniques and establishment of standardized operations have grad- ually raised operation success rate to $98.5 \%$ and reduced major perioperative adverse events to $2.7 \%$ (Boersma et al., 2016).

This study shows that the incidence of pericardial effusion/tamponade in AF patients with CKD was $1.90 \%$. The Asa Plavix Feasibility Study (Reddy et al., 2013) showed that, in patients with anticoagulation contraindications, the incidence of pericardial effusion/tamponade was $3.3 \%$ in the perioperative period. Our study revealed a perioperative mortality rate of $1.10 \%$ after LAAO, which is slightly higher than the previously reported rate in the EWOLUTION study (0.4\%) (Boersma et al., 2017). In all AF patients, the incidence of perioperative stroke was $0.3-2.3 \%$, and that of perioperative bleeding rate was about $1 \%$ (Boersma et al., 2017). Here, we find that the incidence of perioperative stroke and bleeding in $\mathrm{AF}$ patients with CKD was similar to that of general patients. As the application of LAAO 


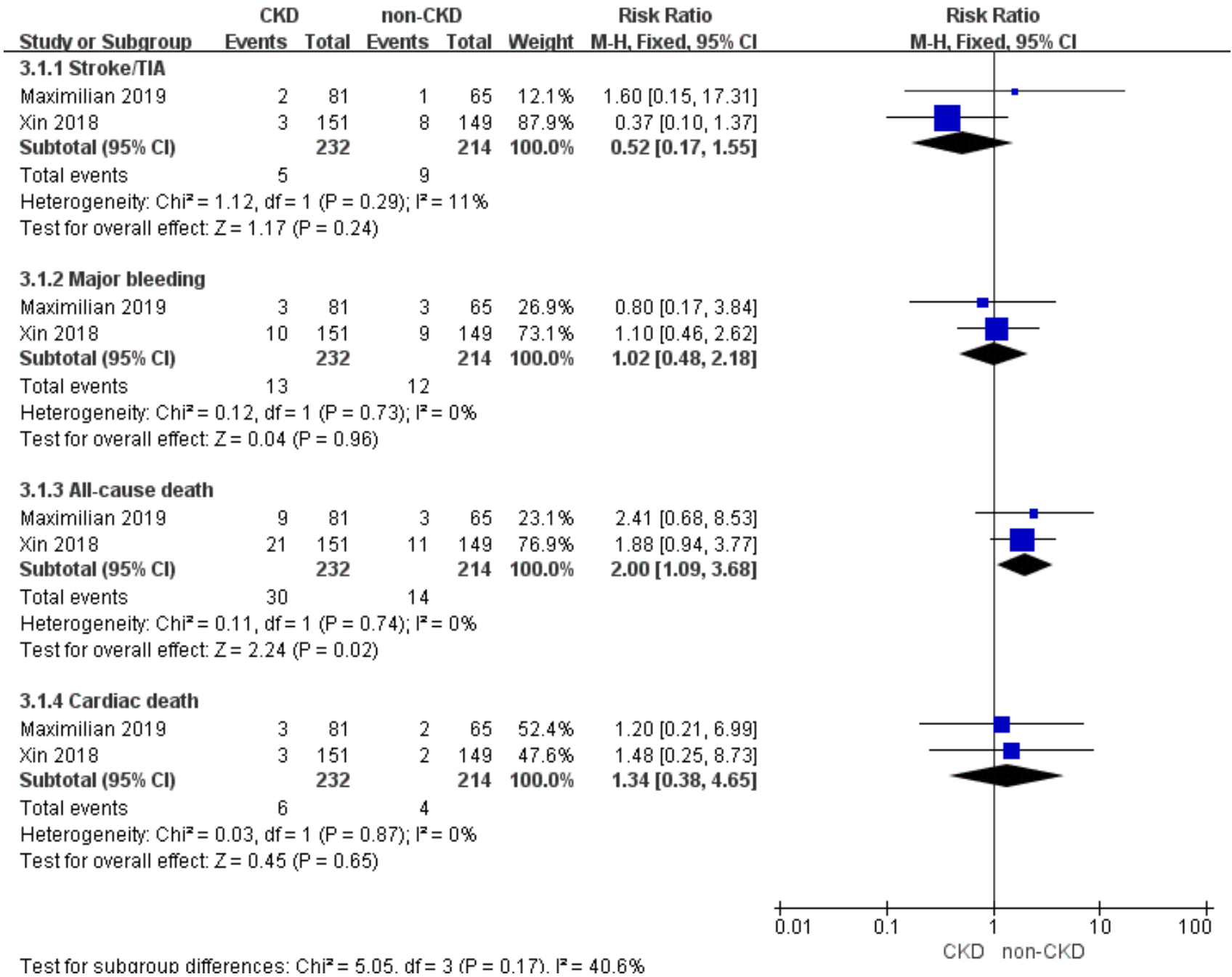

Fig. 5. Forest plots of the meta-analysis comparing the clinical outcomes after left atrial appendage occlusion in patients with or without chronic kidney disease. Patients with CKD exhibited higher all-cause mortality rate after undergoing LAAO (RR:2.00; 95\%CI: 1.09-3.68, $P<0.05)$. There were no significant differences in stroke/TIA (RR: $0.52, P=0.24)$, bleeding (RR: $1.02, P=0.96)$ and cardiac death $(\mathrm{RR}: 1.34, P=0.65)$.

becomes more advanced, the incidence of adverse events in the perioperative period is generally low (Bajwa et al., 2017), even in CKD patients.

Contrast-induced acute kidney injury (CI-AKI) is an important complication resulting from intravascular administration of contrast media, and is likely to occur in CKD patients (Chalikias et al., 2016). Nombela-Franco et al. (Nombela-Franco et al., 2018) reported that incidence of AKI after LAAO was $9 \%$ in general population but lower $(0.8 \%)$ in patients with stage 3 CKD. Of the 905 patients included in this study, only 9 developed CI-AKI. Thus, strategies that improve perioperative management and minimize contrast media dosage are key to avoiding potential deterioration of renal function (Vuddanda et al., 2020).

\subsection{Efficacy and Safety during Follow-up}

The prevalence of AF in CKD patients is $15-20 \%$, which is higher than in the general population (Kirchhof et al., 2016). AF independently increases risk of stroke by 4-5-fold (Zhang et al., 2019). Oral anticoagulants reduce risk of stroke by about twothirds in AF patients (Mtwesi and Amit, 2019). Warfarin is still the first-choice drug for the prevention of stroke in many types of AF (January et al., 2019). However, it interacts with food or other drugs, hence the international normalized ratio (INR) needs to be frequently monitored. Non-vitamin $\mathrm{K}$ antagonists of oral anticoagulants (NOACs) exhibit limited interaction with food and do not require frequent INR monitoring (La-Viola and Guerra, 2018). However, all NOACs undergo kidney clearance and are influenced by glomerular filtration rate (Shin et al., 2018). In AF patients with $\mathrm{CKD}$, the annual stroke/TIA rate following NOAC and warfarin is $1.5-2.5 \%$ and $2-3 \%$, respectively. Additionally, oral anticoagulants results in higher risk of bleeding (3-7\%) in CKD patients than in non-CKD patients (about 3\%) (Diener et al., 2010; Fox et al., 2011; Hohnloser et al., 2012).

The EWOLUTION trial showed that patients with a WATCHMAN LAAO device do not need oral anticoagulants and can be put on a single antiplatelet therapy or no therapy (Boersma et al., 2019). For AF patients fitted with LAAO devices who are contraindicated for anticoagulants, aspirin and clopidogrel use results in an annual incidence of stroke/embolism of $2.3 \%$ (Reddy et al., 2013). Long-term follow-up of the PREVAIL and PROTECT AF 
trials showed that the LAAO group had significantly lower cardiovascular deaths (HR: $0.59, P<0.01$ ), disabling/fatal stroke (HR: $0.45, P=0.05$ ), hemorrhagic stroke (HR: $0.20, P<0.01$ ) and major bleeding (HR: $0.48, P<0.01$ ) relative to those on warfarin (Reddy et al., 2017). The PRAGUE-17 study revealed that LAAO was noninferior to NOAC in preventing ischemic/bleeding (HR: 0.84, $P=0.004$ ) (Godino et al., 2020). Our data suggest that in CKD patients, the incidence of stroke/TIA $(2.17 \%)$ or bleeding $(4.53 \%)$ is low during follow-up period, indicating that LAAO is sufficiently safe and effective.

In this meta-analysis, the all-cause mortality was $12.28 \%$ in CKD patients and cardiogenic factors $(3.98 \%)$ were the leading cause of death. During follow-up, it was observed that cardiac death was caused by heart failure and not atrial appendage occlusion devices (Kefer et al., 2016). The all-cause mortality rate when oral anticoagulants were used to prevent embolic events in AF patients with CKD stage 3 was 5-6\% (Kefer et al., 2016), but it was close to $10 \%$ in $\mathrm{AF}$ patients with end stage renal disease (Siontis et al., 2018). Here, we find that the all-cause mortality in CKD patients was significantly higher than that of non-CKD patients, but the incidence of stroke, bleeding and cardiac death was comparable between the groups. It is worth noting that patients with eGFR of $<30 \mathrm{~mL} / \mathrm{min} / 1.73 \mathrm{~m}^{2}$ had higher mortality rates relative to those with eGFR $>30 \mathrm{~mL} / \mathrm{min} / 1.73 \mathrm{~m}^{2}$ reported previously (Kefer et al., 2016). In this study, many patients had eGFR $<30 \mathrm{~mL} / \mathrm{min} / 1.73 \mathrm{~m}^{2}$, which may account for the higher all-cause mortality rate. Most of the trials included in this study (6/7) did not provide detailed information on renal function, hence it was not analyzed in detail.

\section{Conclusions}

LAAO is safe for AF patients with CKD, including during the perioperative period and long-term follow-up. In cases where surgery is needed in future, discontinuation of anticoagulants does not complicate the process. Advances in LAAO technology and standardization of surgical operations have made this technique ideal for AF patients who are contraindicated for oral anticoagulants.

\section{Study limitations}

Given that no RCTs has directly compared LAAO to oral anticoagulants in AF patients with CKD, we could not make direct comparisons. Most of the trials included in this study did not provide detailed information on renal function, hence it was not analyzed in detail.

\section{Authors' contributions}

Haifu Zhang, Qinxia Zhang, Xingwei Zhang contributed to the conception of the study; Haifu Zhang, Qinxia Zhang, Dong Yang contributed significantly to literature search and data extraction; Haifu Zhang, Qinxia Zhang, Yuanyuan Zhang performed the data analyses and wrote the manuscript; Yuanyuan Zhang, Zhao Xu, Qibin Jiao, Xingwei Zhang helped perform the analysis with constructive discussions.

\section{Funding}

This study was funded by Natural Science Foundation of Zhejiang Province of China (Grant number: LQ18H020006),
Health and Technology Plan of Hangzhou City (Grant number: 0020190126), Health and Technology Plan of Hangzhou City (Grant number: 2017ZD02).

\section{Acknowledgments}

Thanks to all the peer reviewers and editors for their opinions and suggestions.

\section{Conflict of interest}

The authors declare no conflict of interest.

Submitted: April 15, 2020

Received: July 24, 2020

Accepted: July 27, 2020

Published: September 30, 2020

\section{References}

Akagi, Y., Chiba, T., Uekusa, S., Kato, H., Yamamura, S., Aoki, Y., Enoki, M., Ogawara, Y., Kasahara, T., Kimura, Y., Shimizu, T., Takeishi, A., Nakajima, Y., Kobayashi, H. and Sugi, K. (2019) Retrospective cohort study of the efficacy and safety of dabigatran: real-life dabigatran use including very low-dose $75 \mathrm{mg}$ twice daily administration. Journal of Pharmaceutical Health Care and Sciences 5, 17.

Bajwa, R. J., Kovell, L., Resar, J. R., Arbab-Zadeh, A., Mandal, K., Calkins, H. and Berger, R. D. (2017) Left atrial appendage occlusion for stroke prevention in patients with atrial fibrillation. Clinical Cardiology 40, 825-831.

Black-Maier, E., Piccini, J. P. and Granger, C. B. (2019) Left atrial appendage closure: A therapy uniquely suited for specific populations of patients with atrial fibrillation. Journal of Cardiovascular Electrophysiology 30, 2968-2976.

Boersma, L. V. A., Schmidt, B., Betts, T. R., Sievert, H., Tamburino, C., Teiger, E., Pokushalov, E., Kische, S., Schmitz, T., Stein, K. M. and Bergmann, M. W. (2016) Implant success and safety of left atrial appendage closure with the WATCHMAN device: peri-procedural outcomes from the EWOLUTION registry. European Heart Journal 37, 2465-2474.

Boersma, L. V., Ince, H., Kische, S., Pokushalov, E., Schmitz, T., Schmidt, B., Gori, T., Meincke, F., Protopopov, A. V., Betts, T., Foley, D., Sievert, H., Mazzone, P., De Potter, T., Vireca, E., Stein, K. and Bergmann, M. W. (2017) Efficacy and safety of left atrial appendage closure with WATCHMAN in patients with or without contraindication to oral anticoagulation: 1-Year follow-up outcome data of the EWOLUTION trial. Heart Rhythm 14, 1302-1308.

Boersma, L. V., Ince, H., Kische, S., Pokushalov, E., Schmitz, T., Schmidt, B., Gori, T., Meincke, F., Protopopov, A. V., Betts, T., Mazzone, P., Foley, D., Grygier, M., Sievert, H., De Potter, T., Vireca, E., Stein, K., Bergmann, M. W. and following investigators and institutions participated in the EWOLUTION study (2019) Evaluating RealWorld Clinical Outcomes in Atrial Fibrillation Patients Receiving the WATCHMAN Left Atrial Appendage Closure Technology. Circulation: Arrhythmia and Electrophysiology 12, e006841.

Bohula, E. A., Giugliano, R. P., Ruff, C. T., Kuder, J. F., Murphy, S. A., Antman, E. M. and Braunwald, E. (2016) Impact of renal function on outcomes with edoxaban in the ENGAGE AF-TIMI 48 trial. Circulation 134, 24-36.

Brockmeyer, M., Wolff, G., Krieger, T., Lin, Y., Karathanos, A., Afzal, S., Zeus, T., Westenfeld, R., Polzin, A., Heinen, Y., Perings, S., Kelm, M., Medical Faculty, CARID - Cardiovascular Research Institute Düsseldorf, Heinrich-Heine-University, Düsseldorf, Germany and Schulze, V. (2019) Kidney function stratified outcomes of percutaneous left atrial appendage occlusion in patients with atrial fibrillation and high bleeding risk. Acta Cardiologica 18, 1-9.

Chalikias, G., Drosos, I. and Tziakas, D. N. (2016) Prevention of contrastinduced acute kidney injury: an update. Cardiovascular Drugs and Therapy 30, 515-524. 
Coleman, C. I., Evidence-Based Practice Center, Hartford Hospital, Hartford, CT, USA, Kreutz, R., Sood, N., Bunz, T. J., Meinecke, A., Eriksson, D., Baker, W. L. and Evidence-Based Practice Center, Hartford Hospital, Hartford, CT, USA (2019) Rivaroxaban's impact on renal decline in patients with nonvalvular atrial fibrillation: a US marketscan claims database analysis. Clinical and Applied ThrombosisHemostasis 25, 107602961986853.

Dalia, A. A., Kuo, A., Vanneman, M., Crowley, J., Elhassan, A. and Lai, Y. (2020) Anesthesiologists Guide to the 2019 AHAACCHRS focused update for the management of patients with atrial fibrillation. Journal of Cardiothoracic and Vascular Anesthesia 34, 1925-1932.

Della Rocca, D. G., Gasperetti, A., Fassini, G., Mohanty, S., Trivedi, C., Natale, V. N., Horton, R., Gianni, C., Al-Ahmad, A. and Burkhardt, J. D. J. C. (2018) Safety and clinical effectiveness of left atrial appendage occlusion in patients with chronic kidney disease.Circulation 138, A12696-A12696.

Diener, H., Connolly, S. J., Ezekowitz, M. D., Wallentin, L., Reilly, P. A., Yang, S., Xavier, D., Di Pasquale, G. and Yusuf, S. (2010) Dabigatran compared with warfarin in patients with atrial fibrillation and previous transient ischaemic attack or stroke: a subgroup analysis of the RE-LY trial. The Lancet Neurology 9, 1157-1163.

Fox, K. A. A., Piccini, J. P., Wojdyla, D., Becker, R. C., Halperin, J. L., Nessel, C. C., Paolini, J. F., Hankey, G. J., Mahaffey, K. W., Patel, M. R., Singer, D. E. and Califf, R. M. (2011) Prevention of stroke and systemic embolism with rivaroxaban compared with warfarin in patients with non-valvular atrial fibrillation and moderate renal impairment. European Heart Journal 32, 2387-2394.

Genovesi, S., Slaviero, G., Porcu, L., Casu, G., Bertoli, S., Sagone, A., Pieruzzi, F., Rovaris, G., Buskermolen, M., Danna, P., Montoli, A., Oreglia, J., Contaldo, G. and Mazzone, P. (2018) Implant success and safety of left atrial appendage occlusion in end stage renal disease patients: Peri-procedural outcomes from an Italian dialysis population. International Journal of Cardiology 262, 38-42.

Godino, C., Melillo, F., Bellini, B., Mazzucca, M., Pivato, C. A., Rubino, F., Figini, F., Mazzone, P., Della Bella, P., Margonato, A., Colombo, A. and Montorfano, M. (2020) Percutaneous left atrial appendage closure versus non-vitamin $\mathrm{K}$ oral anticoagulants in patients with non-valvular atrial fibrillation and high bleeding risk. Eurointervention 15, 15481554.

Hohnloser, S. H., Hijazi, Z., Department of Medical Sciences, Cardiology, Uppsala University, Uppsala, Sweden, Thomas, L., Alexander, J. H., Amerena, J., Hanna, M., Keltai, M., Lanas, F., Lopes, R. D., Lopez-Sendon, J., Granger, C. B. and Wallentin, L. (2012) Efficacy of apixaban when compared with warfarin in relation to renal function in patients with atrial fibrillation: insights from the ARISTOTLE trial. European Heart Journal 33, 2821-2830.

Holmes, D. R., Reddy, V. Y., Turi, Z. G., Doshi, S. K., Sievert, H., Buchbinder, M., Mullin, C. M. and Sick, P. (2009) Percutaneous closure of the left atrial appendage versus warfarin therapy for prevention of stroke in patients with atrial fibrillation: a randomised non-inferiority trial. The Lancet 374, 534-542.

January, C. T., Wann, L. S., Calkins, H., Chen, L. Y., Cigarroa, J. E., Cleveland, J. C., Ellinor, P. T., Ezekowitz, M. D., Field, M. E., Furie, K. L., Heidenreich, P. A., Murray, K. T., Shea, J. B., Tracy, C. M. and Yancy, C. W. (2019) 2019 AHAACCHRS focused update of the 2014 AHAACCHRS guideline for the management of patients with atrial fibrillation: A Report of the American College of CardiologyAmerican Heart Association Task Force on Clinical Practice Guidelines and the Heart Rhythm Society in collaboration with the Society of Thoracic Surgeons. Circulation 140, e125-e151.

Kefer, J., Tzikas, A., Freixa, X., Shakir, S., Gafoor, S., Nielsen-Kudsk, J. E., Berti, S., Santoro, G., Aminian, A., Landmesser, U., Nietlispach, F., Ibrahim, R., Danna, P. L., Benit, E., Budts, W., Stammen, F., De Potter, T., Tichelbäcker, T., Gloekler, S., Kanagaratnam, P., Costa, M., Cruz-Gonzalez, I., Sievert, H., Schillinger, W., Park, J., Meier, B. and Omran, H. (2016) Impact of chronic kidney disease on left atrial appendage occlusion for stroke prevention in patients with atrial fibrillation. International Journal of Cardiology 207, 335-340.

Kirchhof, P., Benussi, S., Kotecha, D., Ahlsson, A., Atar, D., Casadei, B.,
Castella, M., Diener, H. C., Heidbuchel, H., Hendriks, J., Hindricks, G., Manolis, A. S., Oldgren, J., Popescu, B. A., Schotten, U., Van Putte, B., Vardas, P. and ESC Scientific Document Group (2016) 2016 ESC Guidelines for the management of atrial fibrillation developed in collaboration with EACTS. European Heart Journal 37, 1359-1469.

Kreidieh, B., Rojas, F., Schurmann, P., Dave, A. S., Kashani, A., Rodríguez-Mañero, M. and Valderrábano, M. (2015) Left atrial appendage remodeling after lariat left atrial appendage ligation. Circulation: Arrhythmia and Electrophysiology 8, 1351-1358.

La-Viola, B. and Guerra, C. G. (2018) Risky business: judging the use of non-vitamin $\mathrm{K}$ antagonist oral anticoagulants for non-valvular atrial fibrillation in patients with renal dysfunction. Journal of Atrial Fibrillation 11, 2017.

Levey, A. S., de Jong, P. E., Coresh, J., Nahas, M. E. L., Astor, B. C., Matsushita, K., Gansevoort, R. T., Kasiske, B. L. and Eckardt, K. (2011) The definition, classification, and prognosis of chronic kidney disease: a KDIGO Controversies Conference report. Kidney International 80, $17-28$

Luani, B., Genz, C., Herold, J., Mitrasch, A., Mitusch, J., Wiemer, M., Schmeißer, A., Braun-Dullaeus, R. C. and Rauwolf, T. (2019) Cerebrovascular events, bleeding complications and device related thrombi in atrial fibrillation patients with chronic kidney disease and left atrial appendage closure with the WATCHMAN ${ }^{\mathrm{TM}}$ device. BMC Cardiovascular Disorders 19, 112.

Malyszko, J., Lopatowska, P., Mlodawska, E., Musialowska, D., Malyszko, J. S. and Tomaszuk-Kazberuk, A. (2018) Atrial fibrillation in kidney transplant recipients: is there a place for the novel drugs? Nephrology Dialysis Transplantation 33, 1304-1309.

Mtwesi, V. and Amit, G. (2019) Stroke prevention in atrial fibrillation. Medical Clinics of North America 103, 847-862.

Noble, R. and Taal, M. W. (2019) Epidemiology and causes of chronic kidney disease. Medicine 47, 562-566.

Nombela-Franco, L., Rodés-Cabau, J., Cruz-Gonzalez, I., Freixa, X., Asmarats, L., Gutiérrez, H., Sahay, S., Rodriguez-Gabella, T., MorenoSamos, J. C., Tirado-Conte, G., Goncalves-Ramirez, L. R., RamaMerchan, J. C., Amat-Santos, I. J., O’Hara, G., Martín-Yuste, V., Bethencourt, A., Jimenez-Quevedo, P. and Macaya, C. (2018) Incidence, predictors, and prognostic value of acute kidney injury among patients undergoing left atrial appendage closure. JACC: Cardiovascular Interventions 11, 1074-1083.

Osmancik, P., Tousek, P., Herman, D., Neuzil, P., Hala, P., Stasek, J., Haman, L., Kala, P., Poloczek, M., Branny, M., Chovancik, J., Cervinka, P., Holy, J., Vancura, V., Rokyta, R., Taborsky, M., Kovarnik, T., Zemanek, D., Peichl, P., Haskova, S., Jarkovsky, J. and Widimsky, P. (2017) Interventional left atrial appendage closure vs novel anticoagulation agents in patients with atrial fibrillation indicated for long-term anticoagulation (PRAGUE-17 study). American Heart Journal 183, 108-114.

Potpara, T. S., Ferro, C. J. and Lip, G. Y. H. (2018) Use of oral anticoagulants in patients with atrial fibrillation and renal dysfunction. Nature Reviews Nephrology 14, 337-351.

Reddy, V. Y., Doshi, S. K., Kar, S., Gibson, D. N., Price, M. J., Huber, K., Horton, R. P., Buchbinder, M., Neuzil, P., Gordon, N. T. and Holmes, D. R. (2017) 5-year outcomes after left atrial appendage closure. Journal of the American College of Cardiology 70, 2964-2975.

Reddy, V. Y., Möbius-Winkler, S., Miller, M. A., Neuzil, P., Schuler, G., Wiebe, J., Sick, P. and Sievert, H. (2013) Left atrial appendage closure with the watchman device in patients with a contraindication for oral anticoagulation. Journal of the American College of Cardiology 61, 2551-2556.

Shamseer, L., Moher, D., Clarke, M., Ghersi, D., Liberati, A., Petticrew, M., Shekelle, P., Stewart, L. A. and the PRISMA-P Group (2015) Preferred reporting items for systematic review and meta-analysis protocols (PRISMA-P) 2015: elaboration and explanation. BMJ 349, g7647-g7647.

Shin, J., Secora, A., Alexander, G. C., Inker, L. A., Coresh, J., Chang, A. R. and Grams, M. E. (2018) Risks and benefits of direct oral anticoagulants across the spectrum of GFR among incident and prevalent patients with atrial fibrillation. Clinical Journal of the American Soci- 
ety of Nephrology 13, 1144-1152.

Siontis, K. C., Dr Siontis' current affiliation is the Department of Cardiovascular Medicine, Mayo Clinic, Rochester, MN., Zhang, X., Eckard, A., Bhave, N., Schaubel, D. E., He, K., Tilea, A., Stack, A. G., Balkrishnan, R., Yao, X., Department of Health Sciences Research, Division of Health Care Policy and Research (X.Y., N.D.S.), Mayo Clinic, Rochester, MN., Noseworthy, P. A., Department of Cardiovascular Medicine (P.A.N.), Mayo Clinic, Rochester, MN., Shah, N. D., Department of Health Sciences Research, Division of Health Care Policy and Research (X.Y., N.D.S.), Mayo Clinic, Rochester, MN., Saran, R., Kidney Epidemiology and Cost Center, School of Public Health (A.E., K.H., R.S.), University of Michigan, Ann Arbor., Nallamothu, B. K. and Institute for Healthcare Policy and Innovation (B.K.N.), University of Michigan, Ann Arbor. (2018) Outcomes associated with apixaban use in patients with end-stage kidney disease and atrial fibrillation in the United States. Circulation 138, 1519-1529.

So, C. Y., Cheung, G. S. H., Chan, C. K. Y., Kam, K. K. H., Chan, A. K. Y., Au, S. S. W., Chan, S. K. C., Lam, Y. Y., Lee, A. P. W. and Yan, B. P. Y. (2018) Effect of chronic kidney disease on left atrial appendage occlusion outcome: a single-centre retrospective analysis. Journal of the American College of Cardiology 71, A302.

Tomson, C. and Duffy, S. (2019) Management of chronic kidney disease. Medicine 47, 567-575.

Vuddanda, V. L. K., Turagam, M. K., Umale, N. A., Shah, Z., Lakkireddy, D. R., Bartus, K., McCausland, F. R., Velagapudi, P., Mansour, M. and Heist, E. K. (2020) Incidence and causes of in-hospital outcomes and 30-day readmissions after percutaneous left atrial appendage closure: A US nationwide retrospective cohort study using claims data. Heart Rhythm 17, 374-382.

Xue, X., Jiang, L., Duenninger, E., Muenzel, M., Guan, S., Fazakas, A., Cheng, F., Illnitzky, J., Keil, T. and Yu, J. (2018) Impact of chronic kidney disease on Watchman implantation: experience with 300 consecutive left atrial appendage closures at a single center. Heart and Vessels 33, 1068-1075.

Zhang, L., Steckman, D. A., Adelstein, E. C., Schulman-Marcus, J., Loka, A., Mathew, R. O., Venditti, F. J. and Sidhu, M. S. (2019) Oral anticoagulation for atrial fibrillation thromboembolism prophylaxis in the chronic kidney disease population: the State of the Art in 2019. Cardiovascular Drugs and Therapy 33, 481-488. 